\title{
Neue Aufgabenkultur für digitale Kartenarbeit im Schulunterricht mittels mobiler Geräte
}

\author{
Pascal Tschudi ${ }^{a, b *}$ \\ ${ }^{a}$ Inst.f. Kartografie u. Geoinform. der ETH Zürich, Pascal Tschudi-tschudip@ethz.ch \\ ${ }^{b}$ Lehrerbildung Geografie Sek. I der Pädagogischen Hochschule Zürich, Pascal Tschudi-pascal.tschudi@phzh.ch \\ * Corresponding author
}

Keywords: Schulunterricht, digitale Karten, Kartenarbeit, Aufgabenkultur, Didaktik, digitale Lehrmittel

\begin{abstract}
:
Das gesellschaftliche Leben wird immer digitaler, demzufolge auch die allägliche Lebenswelt von Lernenden. Um mit digitalen Medien angemessen, zielorientiert und reflektiert umgehen zu können, sind entsprechende Kompetenzen gefordert. Im Fachbereich der Geografie, insbesondere in der Fähigkeit der räumlichen Orientierung, besitzen digitale Orientierungsmittel einen zentralen Stellenwert und deshalb wird dessen Einsatz im Unterricht auch in der Ausbildung von Lehrpersonen fachdidaktisch gefördert. Digitale Karten ermöglichen eine Ersatzbegegnung mit dem Lehrgegenstand oder finden beim ausserschulischem Lernen Einsatz, sie motivieren zur Erschliessung der Lerninhalte und sie bieten eine Diskussionsgrundlage. Des Weiteren unterstützen sie kooperative und handlungsorientierte Lernformen, nebst möglichen Individualisierungen und Differenzierungen in heterogenen Klassenverbänden.
\end{abstract}

Gedruckte Atlanten werden schon seit geraumer Zeit im Schulkontext erfolgreich eingesetzt, insofern sind zu traditionellen Papierkarten passende Frage- und Problemstellungen, methodische Vorgehensweisen und konstruierte Aufgabenstellungen ausreichend didaktisch fundiert. Nun steht aber mit dem Wechsel des Mediums von der analogen zur digitalen Welt ein Neudenken der Aufgabenkultur zur Förderung und Forderung der räumlichen Orientierungskompetenz an. Die praktische Kartenarbeit, welche die drei Teilkompetenzen Karten «anfertigen», «auswerten» und «bewerten» gleichermassen berücksichtigen soll, verändert sich wesentlich durch Einsatz digitaler Lehrmittel.

In diesem Vortrag wird veranschaulicht, wie Karten aus der Druckausgabe des Schweizer Weltatlas durch eine mobile App für Tablets mit vielfältigen digitalen Darstellungen und Funktionalitäten aufgewertet werden können.
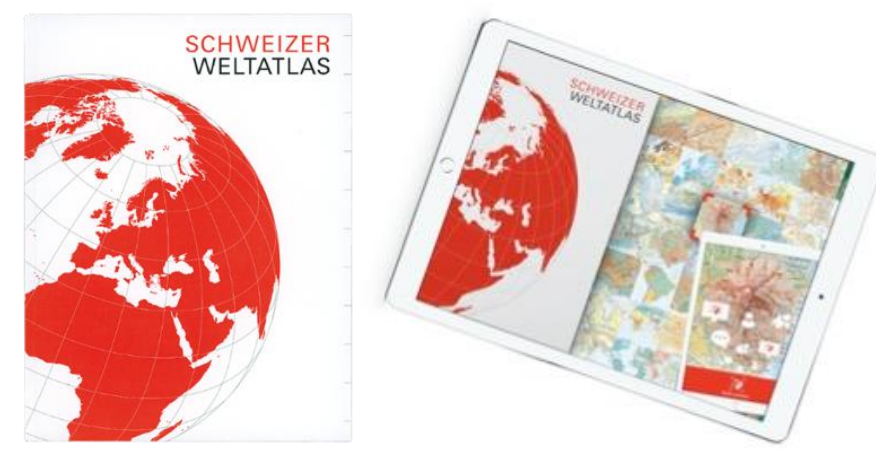

Abbildung 1. links: Druckausgabe Schweizer Weltatlas rechts: Prototyp SWA-App

Einerseits soll ein bereits existierender Prototyp aufzeigen, wie dies technisch-kartografisch mit der Entwicklungsumgebung Unity umgesetzt werden könnte. Dank Kombination mit der Bilderkennungsoftware Vuforia kann der Zugang zu den digitalen Abbildungen durch Scannen der entsprechenden Karte aus der Druckausgabe mit der Kamera des mobilen Endgerätes intuitiv und einfach gestaltet werden. Anschliessend können auf einer digitalen 1:1 Kopie der Papierkarte Lehrpersonen und Lernende gleichermassen bestehende Inhalte markieren, hervorheben und zeichnerisch ergänzen oder aber neue Sachverhalte eintragen. Ausserdem können je nach Frage- oder Problemstellung, die es zu erarbeiten gilt, thematische Kartenebenen ein- und ausgeblendet werden. Damit Karten noch einfacher zu Vergleichszwecken herbeigezogen werden können, um weitergehende Zusammenhänge zu analysieren, bietet die App entsprechende Swipe- oder Überlagerungswerkzeuge. In geografischen Räumen, wo die Topografie einen 
entscheidenden Einfluss auf physisch- und human-geografische Sachverhalte besitzt, können Kartenbilder dreidimensional als Blockbild dargestellt und manipuliert werden. Damit bei grossmassstäbigen interregionalthematischen Karten durch verzerrende Projektionen keine Fehlvorstellungen bei den Lernenden entstehen, können diese Erdkarten auf einen virtuellen 3D-Globus projiziert werden. Schliesslich ermöglicht die App einen vereinfachten Zugang zu einem Onlineangebot mit weiteren Lehr- und Lernmaterialien für die Kartenarbeit. In Planung sind weitere digitale Inhalte, Darstellungen und Funktionen, welche weitestgehend unabhängig vom reinen digitalen Abbild der Papierkarten fungieren (z.B. fachdidaktische Methoden/Medien, Storymaps).

Andererseits soll in diesem Vortrag verdeutlicht werden, wie die inhaltlich-thematischen Lerninhalte nun mit weitergehenden und angepassten Aufgabenstellungen von den Lernenden anderweitig digital erarbeitet werden und so die Kartenkompetenz fördern. Verschiedenste implementierte Funktionalitäten ermöglichen dabei konkrete Handlungen (z.B. Einzeichnen/Markieren, selbst Kartieren, Topografie manipulieren, Höhenstufen extrahieren, Beleuchtungssituationen Sonne-Erde simulieren usw.) und unterstützen den Erkenntnisgewinn zusätzlich. Einige mögliche Aufgabenbeispiele hierfür sind das Kartieren von Rückzugsorten für ruhende Wildtiere im Skigebiet Oberengadin, das Evaluieren von Skianlagen, welche durch den Klimawandel bedroht sind, das Beschreiben von Abflussregimes von Bergbächen im Sarganserland, das Vergleichen der Nutzung der Schwemmebene durch den Menschen im Laufe der Zeit. Oder das Analysieren der Flächenanteile der beiden Bodentypen «Taigaböden» und «Regenwaldböden» auf einer Erdkarte projiziert auf dem virtuellen Globus.
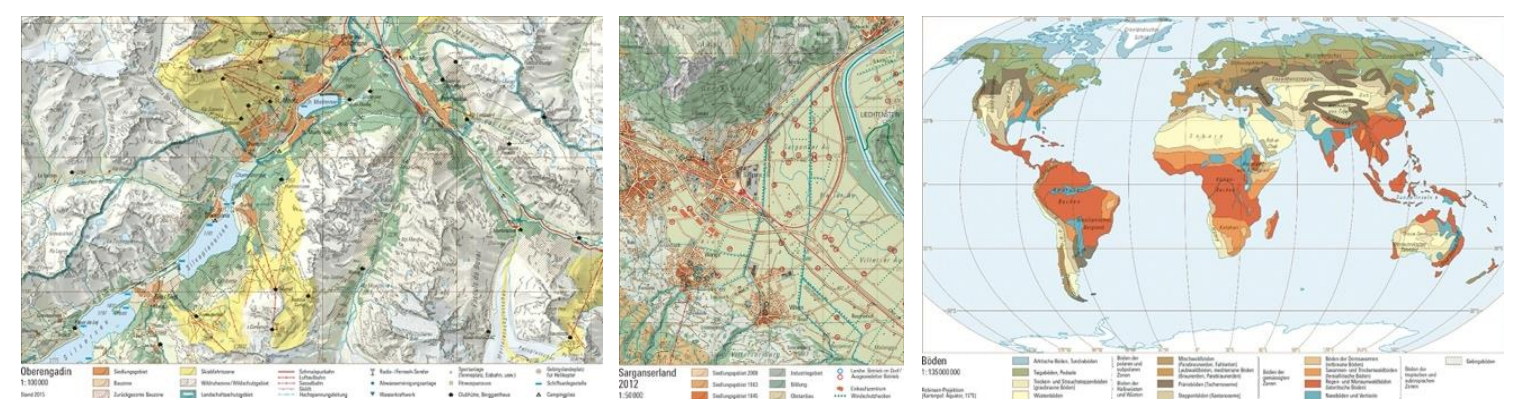

Abbildung 2. Thematische Karten: links: Oberengadin Mitte: Sarganserland rechts: Böden

Neue Möglichkeiten, welche digitale Orientierungsmittel und Karten im Unterrichtseinsatz bieten, müssen einerseits technisch-kartografisch sinnvoll umgesetzt und präsentiert werden. Andererseits können damit neue Frage- und Problemstellungen erarbeitet werden, dazu müssen aber die entsprechenden Aufgabenstellungen neu gedacht werden, damit sie didaktisch wertvoll sind. 